\section{Assessment of the arterial tonometer (N-CAT) for the continuous blood pressure measurement in rapid atrial fibrillation}

The N-CAT is a newly developed arterial tonometer (TBP) able to determine systolic, diastolic and mean arterial blood pressures continuously and noninvasively. The aim of this study was to evaluate the accuracy and reliability of TBP relative to directly measured invasive blood pressure (IBP) in ten haemodynamically stable postoperative cardiac patients who were in rapid atrial fibrillation ( $H R \geq 100 \mathrm{bpm}$ ). There were differences between TBP and IBP for systolic $(-1.7 \mathrm{mmHg})$ and diastolic $(+0.9 \mathrm{mmHg})$ values but not for the mean arterial blood pressures. The N-CAT was able to follow blood pressure changes closely and demonstrated an average systolic, diastolic and mean bias ( $\pm S D$ ) of $-1.71 \pm 4.6,0.99 \pm 4.6$ and 0.33 $\pm 4.2 \mathrm{mmHg}$, respectively. Although these biases are within the required standards for equivalency for noninvasive blood pressure to invasively determined blood pressure, approximately $20 \%$ of the readings were $> \pm 10 \mathrm{mmHg}$ while only $5 \%$ were $> \pm 20 \mathrm{~mm} \mathrm{Hg}$. Moreover, there were occasional discrepancies of sufficient magnitude and duration which may limit the clinical usefulness of the $\mathrm{N}-\mathrm{CAT}$ in patients in whom continuous and accurate blood pressure measurement is required.

Key words

HEART: arrhythmia, fibrillation, atrial;

MEASUREMENT TECHNIQUES: blood pressure; ARRYTHMIA: atrial fibrillation.

From the Department of Anesthesiology, Montreal Heart Institute, 5000 Belanger Street East, Montreal, Quebec HIT 1 C8 Canada.

Address correspondence to: Dr. Norman R. Searle, Département d'Anesthésie-Réanimation, Institut de Cardiologie de Montréal, 5000 Bélanger est, Montréal, Québec HIT 1 C8 Canada.

Accepted for publication 17th December, 1992.
Norman R. Searle MD, Jean Perrault PhD, Hélène Ste-Marie RN, Charles Dupont MSc
Le $N$-CAT est un nouveau moniteur de pression artérielle qui utilise le principe de tonométrie artérielle (TBP) pouvant déterminer la pression systolique, diastolique et moyenne de façon continue et non invasive. Le but de cette étude était d'évaluer la précision et la fiabilité de la TBP par rapport à la pression artérielle invasive (IBP) chez des patients après une chirurgie cardiaque, hémodynamiquement stable mais manifestant une fibrillation auriculaire rapide $(F C>100 \mathrm{batt} / \mathrm{min})$. $l \mathrm{l}$ y avait une différence significative entre la pression systolique (-1.7 $\mathrm{mmHg}$ ) et diastolique ( $+0.9 \mathrm{mmHg}$ ) dérivées par la TBP vis$\grave{a}$-vis IBP sans qu'il y ait de différence pour la pression moyenne entre les deux méthodes. Le $N$-CAT s'est avéré fiable dans sa capacité de suivre les changements de pression artérielle. Ses biais TBP sysolique, diastolique et moyen sont $-1,71 \pm$ $4,6,0,99 \pm 4,6$ et $0,33 \pm 4,2 \mathrm{~mm} \mathrm{Hg}$ respectivement. Ces valeurs de biais répondent aux critères d'équivalence pour une pression invasive. Malgré cela, approximativement $20 \%$ des valeurs de $T B P$ étaient $> \pm 10 \mathrm{mmHg}$ tandis que $5 \%$ étaient $> \pm 20$ mmHg. De plus, il y eut des moments où la différence entre la TBP et la IBP était d'une durée et de magnitude non acceptable, limitant son utilité clinique chez des patients où la mesure de la pression artérielle, de façon exacte et continue, est souhaitable.

Measurement of arterial pressure, either intermittently or continuously, has been standard practice in operating rooms, recovery rooms and intensive care units for decades. If one wants accuracy and beat-to-beat measurement, then direct measurement of intraarterial pressure is used despite some inherent risks with the procedure. However, in those patients in whom there are no absolute indications for invasive arterial pressure, an accurate continuous indirect arterial pressure monitor would be preferable to an invasive technique. Recently, with the advent of microprocessor-based technology, it is possible to re- 

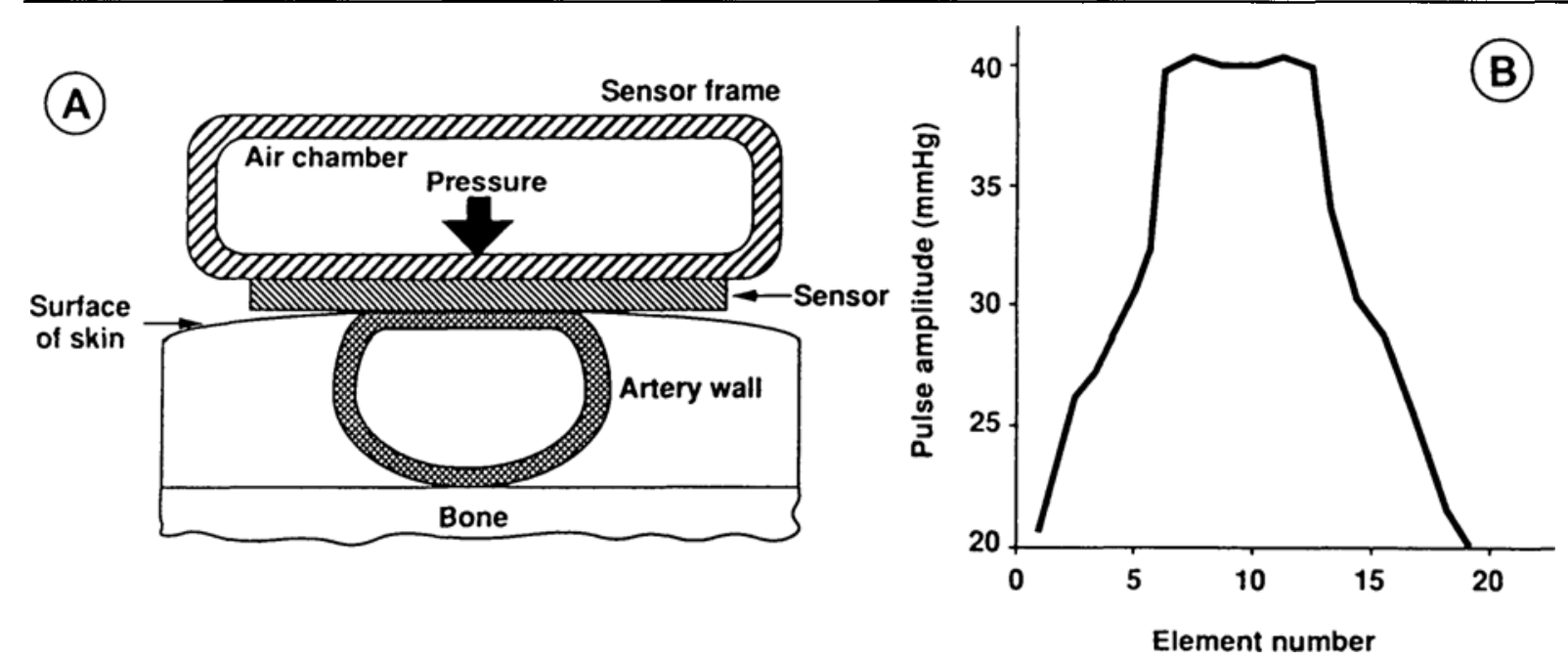

FIGURE I (A) Diagrammatic representation of the tonometric blood pressure attachment. Notice the artery is partially flattened between the sensor and the underlying bone. A series of piezoresistive pressure transducers is mounted in the sensor element. (B) Individual piezoresistive pulsepressure signal from each transducer.

produce noninvasively real-time arterial waveforms in addition to determining systolic, diastolic and mean blood pressure continuously. A new generation of continuous blood pressure monitors, each relying on its own technology, is marketed. One such apparatus is the N-CAT N-500 (Nellcor, Hayward, CA) monitor which relies on arterial tonometry to generate the blood pressure waveform. ${ }^{1}$ Briefly, arterial tonometry consists of a series of 15 piezoresistive pressure transducers mounted on a wrist attachment (Figure 1A). The sensor is brought into contact with the radial artery where sufficient pressure is exerted to flatten it partially. A computer analyses the signals from each piezoresistive pressure transducer and selects the one offering the greatest pulse amplitude (Figure 1B). This pulse amplitude is then converted to a waveform. Methods have yet to be developed to calibrate these pressure waveforms directly against blood pressure. Typically, tonometric blood pressure (TBP) utilizes an alternative method for calibration of the transduced pressure. The N-CAT N-500 has an integrated blood pressure device that relies on the oscillometric technique for calibration.

Little has been published on the performance of TBP in clinical situations. ${ }^{2}$ Acceptability of a monitoring technique for clinical practice is based on both the applicability and performance of the monitor. If this new technology is to replace the existing intermittent noninvasive blood pressure monitor and to reduce the use of an invasive blood pressure technique, it must be accurate and reliable in reflecting direct arterial pressure. Investigations should evaluate these monitors under various pathological states. The aim of this study was to examine the accuracy of TBP in the presence of rapid atrial fibrillation.

\section{Methods}

After Bioethics Committee approval, ten open-heart surgery patients were studied. All were one or two days after surgery and were without distress or complication. Eligibility for the study included rapid atrial fibrillation with heart rate $\geq 100 \mathrm{bpm}$, haemodynamic stability and absence of angina and/or congestive heart failure. Excluded from the study were patients who had different noninvasive blood pressure values on either the left or right side. All patients also had invasive blood pressure (IBP) monitoring with a 20-g Teflon cannula inserted in a radial artery linked to a disposable high-fidelity pressure transducer (Sorenson Transpac, Abbott Critical Care System). The arterial cannula was connected to the pressure transducer with a $180 \mathrm{~cm}$ long, $1.5 \mathrm{~mm}$ internal diameter, low compliance tubing. The arterial transducer was zeroed at the mid-axillary line and the calibration was checked for accuracy and linearity with a mercury manometer following internal electric calibration. The characteristics of the dynamic response of the IBP system was not measured, but using the fast flush technique, no excessive damping or ringing was noticed. ${ }^{3}$ The transducer waveform was displayed on a Siemens 1281 monitor (Siemens Medical Electronics Inc., Danvers, MA) and the cable attachment was verified with a Sorenson Transpac Transducer Simulator (no 42601-01 Abbott Critical Care System).

The contralateral radial artery was used to determine tonometric blood pressure (TBP). The wrist-mounted apparatus was apposed to the radial artery (Figure 1A) and the arterial waveform was reproduced on the N-CAT N-500 monitor. Calibration for TBP was obtained from 
TABLE I Average blood pressure response between the two systems

\begin{tabular}{llll}
\hline & Systolic & Diastolic & Mean \\
\hline Tonometric range & $124.9 \pm 15.0$ & $67.2 \pm 10.9$ & $87.1 \pm 14.7$ \\
& $(95-182)$ & $(31-108)$ & $(54-159)$ \\
Invasive range & $126.6 \pm 15.2$ & $66.4 \pm 8.2$ & $87.0 \pm 10.0$ \\
& $(100-163)$ & $(45-95$ & $(65-110)$ \\
& $P<0.001$ & $P<0.005$ & $P=\mathrm{NS}$ \\
\hline
\end{tabular}

All blood pressure values $(\mathrm{mmHg})$ are expressed as mean $\pm \mathrm{SD}$.

oscillometric blood pressure determinations using an appropriate size blood pressure cuff. Oscillometric blood pressure was determined on the ipsilateral side of the IBP. This calibration was performed every $2.5 \mathrm{~min}$ for three consecutive measurements and then automatically at five-minute intervals.

Each patient was studied for $30 \mathrm{~min}$. For the purpose of the study, each 60 -sec average signal was considered as a one-time sample for comparison of TBP and IBP measurements. Paired values for systolic, diastolic and mean pressure were analyzed by Student's $t$ test for differences between the two blood pressure methods. Also, determination of the accuracy of the N-CAT monitor by measuring its bias (i.e., the tendency to overestimate or underestimate) and precision (i.e., mean absolute error) according to the following formulas:

Bias $=\sum_{i=1}^{n} \frac{\mathrm{TBP}-\mathrm{IBP}}{n} \quad$ Precision $=\sum_{i=1}^{n}\left|\frac{\mathrm{TBP}-\mathrm{IBP}}{n}\right|$

where $i=$ pressure determination and $n=$ number of pressures compared.

Individual bias and precision for systolic, diastolic and mean pressure values were averaged to give the average bias and precision for the N-CAT monitor. Thus, each patient contributed equally to the measurement of the performance of this device.

Scatterplots of paired TBP - IBP differences versus the average of the paired blood pressure measurements for systolic, diastolic and mean pressure were made according to the method of Bland and Altman. ${ }^{4}$

Finally, we calculated the occurrence of two or more consecutive paired measurements during which the difference between TBP and IBP was either $> \pm 10 \mathrm{mmHg}$ or $\pm 20 \mathrm{mmHg}$. These were then expressed as discrepancies per patient-hour for an interval of one to five minutes or $>$ five minutes.

\section{Results}

Measurements were obtained from seven men and three women after cardiac surgery. Their average age, weight, height and heart rate were $60.8 \pm 11.7 \mathrm{yr}, 70.5 \pm 14.8$ $\mathrm{kg}, 166.3 \pm 6.3 \mathrm{~cm}$, and $112.2 \pm 13.2 \mathrm{bpm}$ respectively. A total of 300 pairs of systolic, diastolic and mean TBP and IBP measurements were analysed. Average systolic, diastolic and mean TBP and IBP are presented in Table I. Lower average systolic TBP $(P<0.01)$ and higher diastolic TBP $(P<0.05)$ than the IBP values were found while no difference was seen for mean TBP and IBP blood pressure values.

Individual as well as overall average bias and precision values for the N-CAT monitor are listed in Table II. Single minute averages for TBP and IBP are plotted in Figure 2. Tonometric blood pressure was able to track changes in pressure. Scatterplots of systolic, diastolic and mean TBP minus IBP (TBP - IBP) versus the average of the pressure measured by the two devices (TBP + IBP) 2) are illustrated in Figures 3 to 5 . The $95 \%$ bias intervals $( \pm 2$ SD) are also demarcated in each figure.

The frequency distribution for systolic, diastolic and mean pressures difference between TBP and IBP is shown in Figure 6. Less than 5\% of all the systolic, diastolic and mean blood pressure readings fell in the category of $> \pm 20 \mathrm{mmHg}$ while roughly $20 \%$ of measurements occurred with a $> \pm 10 \mathrm{mmHg}$ difference (Figure 6). The duration and magnitude of blood pressure discrepancies, expressed as discrepancies per patient-hour, are given in Figure 7. The total number of discrepancies was small. Most were of short duration (one to five minutes) and of low magnitude $( \pm 10 \mathrm{mmHg})$. The frequency of discrepancies (TBP - IBP $> \pm 10 \mathrm{mmHg}$ ) for systolic blood pressure lasting between one to five minutes was 1.6 per patient-hour while it was 0.2 per patient-hour for intervals lasting $>$ five minutes. There was no interval of discrepancies that exceeded $\pm 20 \mathrm{mmHg}$ for the mean arterial pressure although the systolic and diastolic blood pressures had, on average, one interval for each 2.5 patient-hour $> \pm 20 \mathrm{mmHg}$.

\section{Discussion}

Recently, several noninvasive blood pressure monitors able to reproduce continuous arterial waveforms have emerged. These monitors are intended for use in operating rooms and possibly in postoperative care units where blood pressure can change rapidly and where direct intraarterial monitoring might not be justified. Before the anaesthetist can consider their use, it is necessary to verify their accuracy with the standard IBP.

Determination of the arterial blood pressure by the invasive method, especially the systolic and diastolic values, also has its limitations. It is dependent on the fidelity of the transduced arterial waveform which may suffer inaccuracies related to the dynamic response of the catheter, tubing and transducer system. Although this study did not assess the dynamic-response characteristics 
TABLE II Individual bias and precision values for systolic, diastolic and mean blood pressures for the N-CAT monitor relative to invasive pressure

\begin{tabular}{|c|c|c|c|c|c|c|}
\hline$P t$ & Syst-bias & Syst-prec & Diast-bias & Diast-prec & Mean-bias & Mean-prec \\
\hline 1 & -7.4 & 7.6 & 1.6 & 3.0 & -0.2 & 1.7 \\
\hline 2 & -10.8 & 12.3 & 4.4 & 6.5 & 0.7 & 4.9 \\
\hline 3 & 0.6 & 5.8 & -3.4 & 6.5 & 2.1 & 5.4 \\
\hline 4 & -0.1 & 5.3 & 2.4 & 6.7 & -0.3 & 5.5 \\
\hline 5 & 0.3 & 5.1 & -2.8 & 10.0 & -2.0 & 7.7 \\
\hline 6 & -3.3 & 3.5 & -1.4 & 2.9 & -4.1 & 4.2 \\
\hline 7 & -4.1 & 5.9 & 11.7 & 11.9 & 1.2 & 5.6 \\
\hline 8 & 2.1 & 8.3 & -2.1 & 7.2 & -2.4 & 6.6 \\
\hline 9 & 3.3 & 9.1 & 1.5 & 10.0 & I0.9 & 11.5 \\
\hline 10 & 2.3 & 8.4 & -2.1 & 7.3 & -2.6 & 7.0 \\
\hline Avg & -1.71 & 7.13 & 0.99 & 7.21 & 0.33 & 6.00 \\
\hline $\mathrm{SD}$ & \pm 4.60 & \pm 2.55 & \pm 4.56 & \pm 2.91 & \pm 4.20 & \pm 2.56 \\
\hline
\end{tabular}

Values are mean for individual patient (Pt) and mean (Avg) \pm standard deviation (SD) for the entire study group. Bias and precision units are in $\mathrm{mmHg}$. Diast $=$ diastolic; Prec $=$ precision; Syst $=$ Systolic.

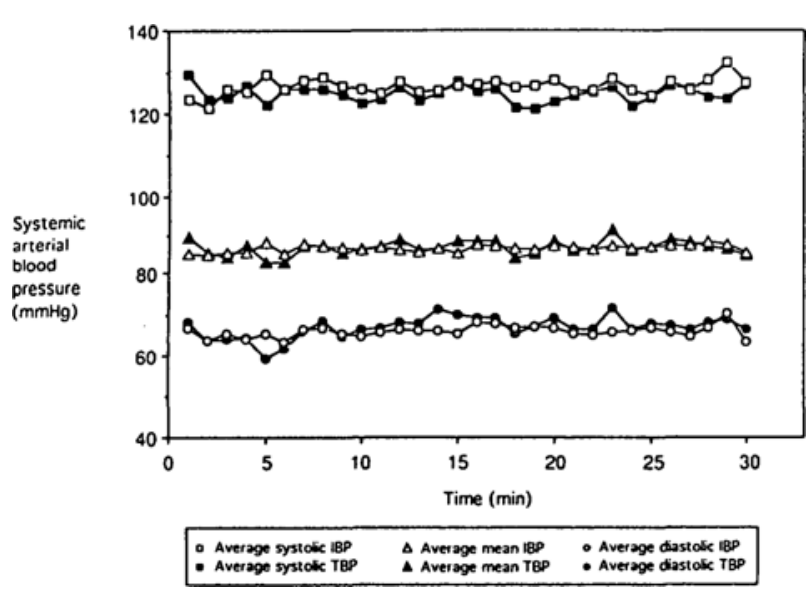

FIGURE 2 Graphic representation of single minute average for systolic, diastolic and mean TBP and IBP.

of the IBP system, the fast flush technique did not reveal excessive ringing. As suggested by Gibbs et al., ${ }^{5}$ to circumvent this possible inaccuracy with systolic and diastolic measurements, one can rely on comparing mean arterial pressure (MAP) values provided that algorithms for its derivation from each monitor are compatible. Using the minute average value, it is unlikely that differences in MAP algorithms contribute to any large discrepancies between N-CAT and IBP measurements.

Another important concern is that comparative studies need to be carried out not only in steady-state conditions, but also in varied clinical situations (rapid blood pressure changes, dysrhythmia, etc.). To date, only the Finapres monitor has seen extensive clinical assessments. ${ }^{5-9}$

The N-CAT N-500 is a newly developed, noninvasive,

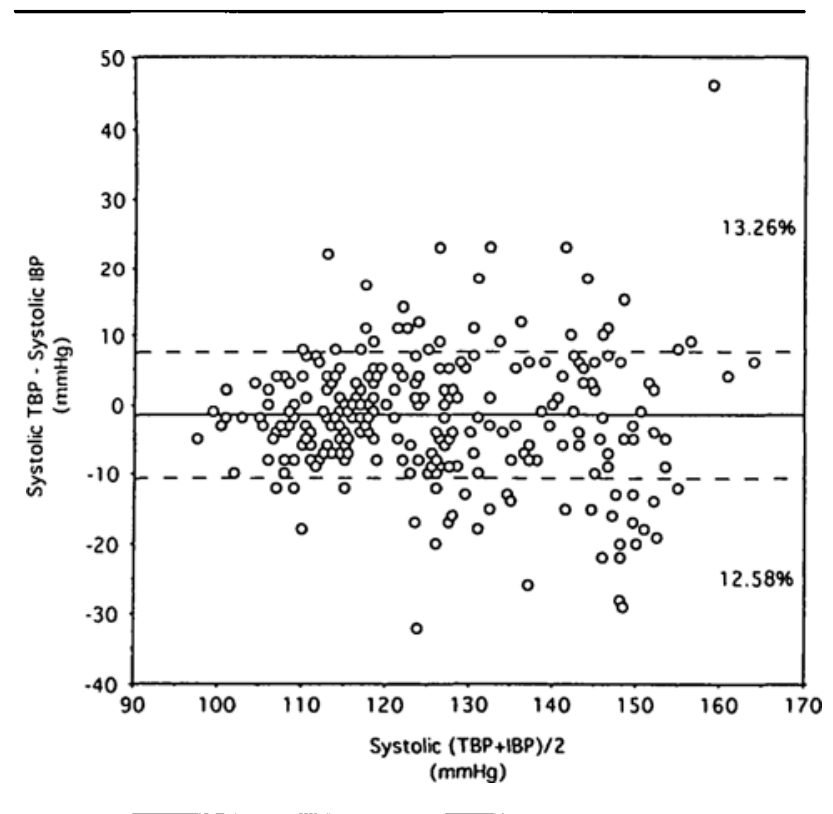

FIGURE 3 Scatterplots of the difference between systolic TBP and IBP (bias) versus the average systolic TBP and IBP values. The average bias line is marked by the solid line. The 2 SD limits of the bias are given by the dotted lines. The percentage value indicated above and below the 2 SD lines represents the number of readings following outside these limits.

real-time, arterial blood pressure monitor, which is able to track rapid blood pressure changes and to reproduce intraarterial recording under steady-state anaesthesia and during controlled hypotension. ${ }^{2}$ Aside from the study by Kemmotsu et al., ${ }^{2}$ tonometric blood pressure monitoring has had few clinical assessments. Because of the frequent occurrence of atrial fibrillation in patients still having invasive monitoring after cardiac surgery, we chose them 


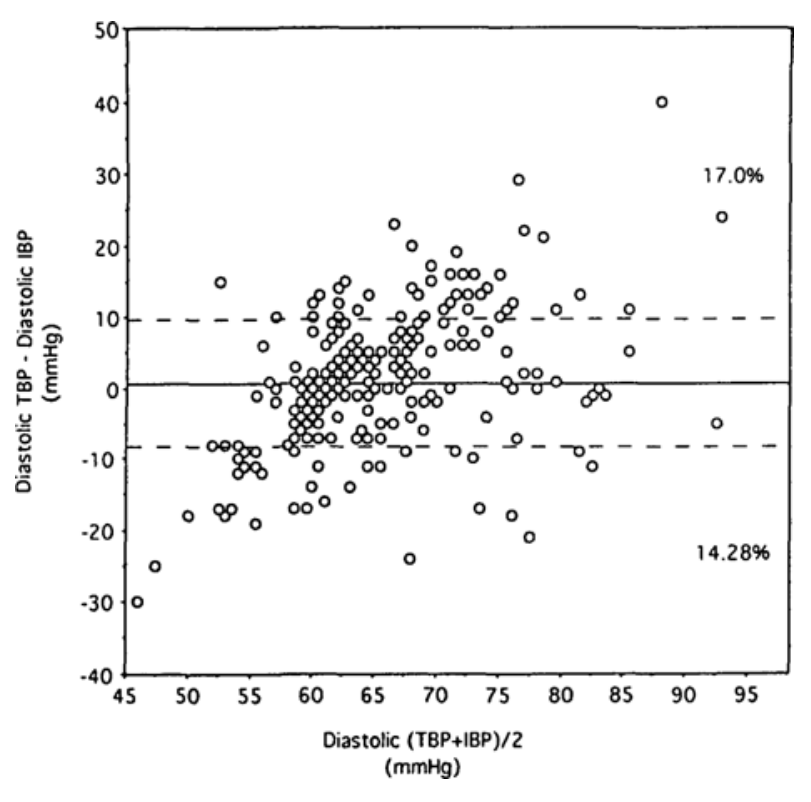

FIGURE 4 Scatterplots of the difference between diastolic TBP and IBP (bias) versus the average diastolic TBP and IBP values. The average bias line is marked by the solid line. The 2 SD limits of the bias are given by the dotted lines. The percentage value indicated above and below the 2 SD lines represents the number of readings following outside these limits.

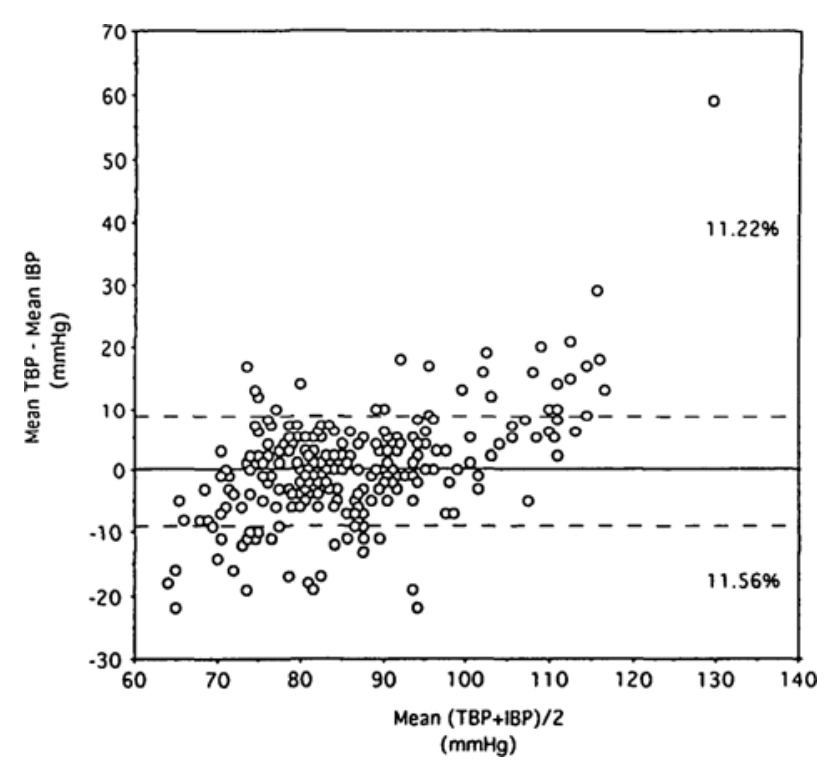

FIGURE 5 Scatterplots of the difference between mean TBP and IBP (bias) versus the average TBP and IBP values. The average bias line is marked by the solid line. The 2 SD limits of the bias are given by the dotted lines. The percentage value indicated above and below the 2 SD lines represents the number of readings following outside these limits.
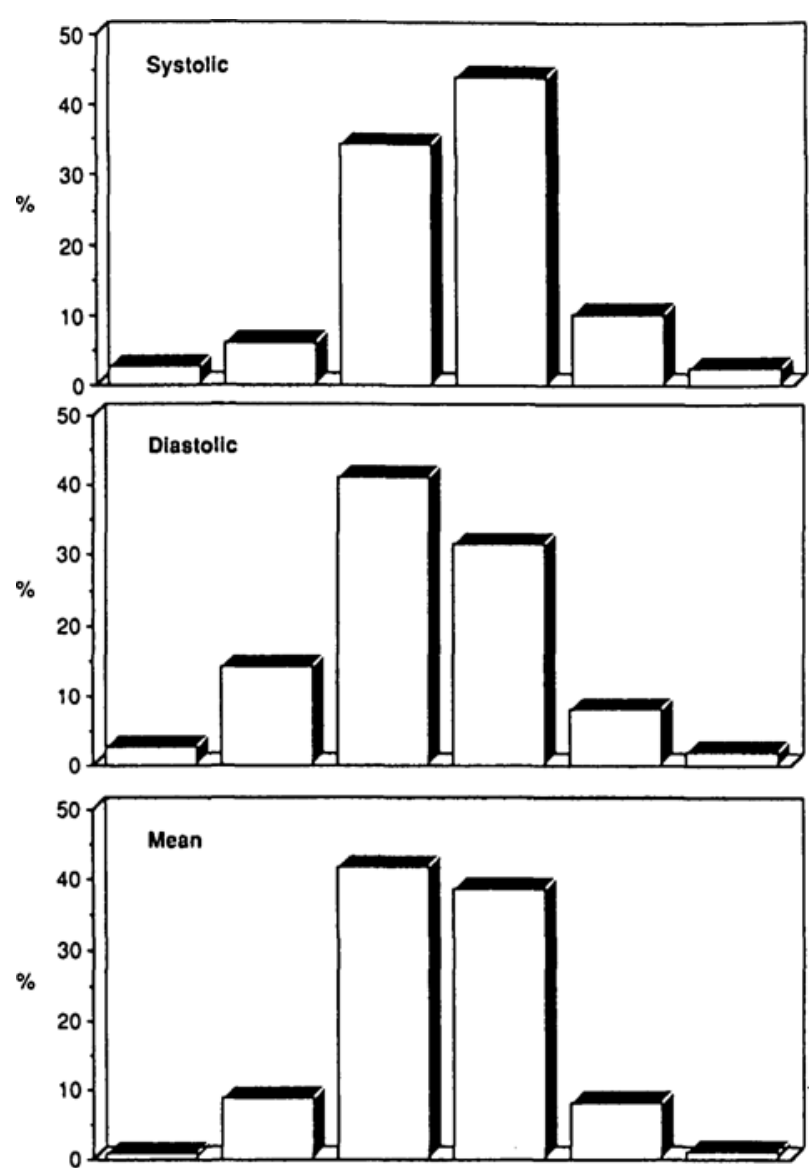

$(<-20)(>-20<-10)(>-10<0)(>0<10)(>10<20) \quad(>+20)$ Difference $\mathrm{mmHg}$

FIGURE 6 Histograms representing the frequency distribution for the difference between systolic, diastolic and mean TBP and IBP.

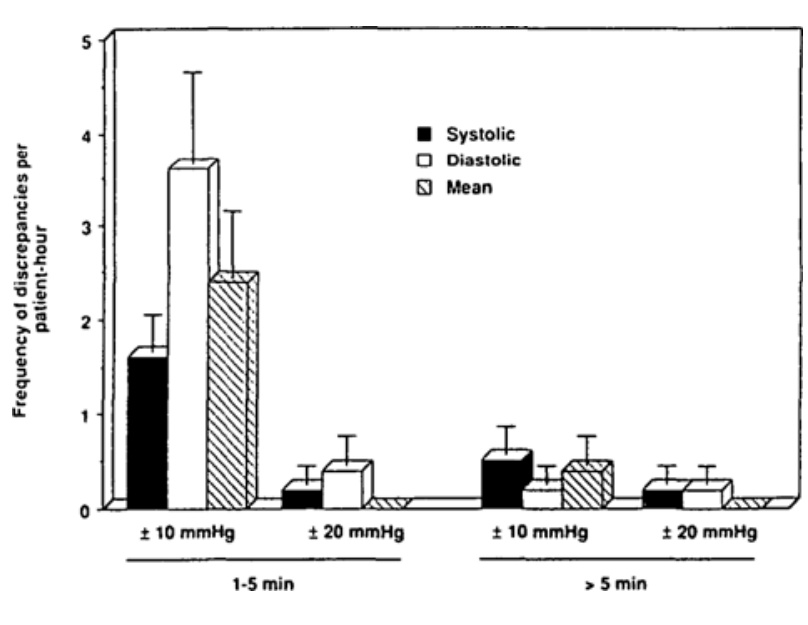

FIGURE 7 The duration of discrepancies per patient-hour between systolic, diastolic and mean TBP and IBP. The histograms indicate mean \pm SEM. 
to evaluate the accuracy and reliability of N-CAT N500 monitor.

This study found a lower systolic TBP (bias - 1.71 \pm 4.6 ), and a higher diastolic TBP (bias $0.99 \pm 4.56$ ) but no difference in average mean blood pressure. According to the accuracy standards of the Association for the Advancement of Medical Instrumentation for equivalence of noninvasive blood pressure devices to invasively determined arterial pressure, the average bias should be within $\pm 5 \mathrm{mmHg}$ and the SD of the bias not $>8 \mathrm{mmHg}$. The N-CAT satisfied the criteria for bias and was able to closely track IBP changes (Figure 2 and Table II). Our average bias results are in agreement with those previously reported. ${ }^{2}$ Even though there were differences in systolic and diastolic blood pressures, changes of this magnitude (i.e., a few millimeters of mercury) may have little or no clinical importance and probably falls within the error of the measuring technique. This lack of clinical importance when comparing only the average TBP and IBP readings and the acceptable average bias values may give a false sense of equivalence between the two methods. It should be pointed out that two of ten patients showed individual bias that fell outside the accepted criteria.

Despite the generally satisfactory performance by the N-CAT, Figures 3 to 5 and Figure 6 identify a number of TBP values that fell outside 2 SD (approximately 10 $\mathrm{mmHg}$ ) from the average bias. This narrow range (average bias $\pm 2 \mathrm{SD}$ ) for assessment of error may be too rigorous. Similarly, it is difficult to state what magnitude of error in blood pressure measurement becomes clinically important. Most anaesthetists would show concern when the range of error becomes $> \pm 10 \mathrm{mmHg}$ and all would agree that errors $> \pm 20 \mathrm{mmHg}$ are unacceptable. Furthermore, the duration of error is also pertinent. The clinical importance of an error that persists $<$ one minute is not comparable with an error that lasts $>$ five minutes. If the duration and magnitude of the error are prolonged, this may lead to inappropriate intraoperative interventions with the potential for increased perioperative morbidity. In expressing the duration and magnitude of a clinically important error $( \pm 10 \mathrm{mmHg})$ or $\pm 20 \mathrm{mmHg}$ ) by the number of discrepancies per patient-hour, Gibbs et al..$^{5}$ wanted to define another important characteristic for the evaluation of a new monitoring technique. Our average discrepancies for the mean arterial pressure were 2.4 and 0.4 per patient-hour $>$ $\pm 10 \mathrm{mmHg}$ for intervals of one to five minutes and $>$ five minutes respectively. There was no interval where the mean arterial blood pressure discrepancies were $>$ $\pm 20 \mathrm{mmHg}$.

Given the limitation of our experimental protocol and the lack of: (1) comparative study on the subject of TBP and (2) appropriate statistical analysis for data of this type, the N-CAT N-500 monitor was able to track fairly accurately the mean arterial blood pressure. Nevertheless large discrepancies $( \pm 20 \mathrm{mmHg})$ with a duration $>$ five minutes for systolic and diastolic TBP measurements occurred at a frequency of 0.2 per patient-hour (Figure 7).

In summary, the N-CAT N-500 monitor represents a considerable advance in noninvasive BP determination. Presently, it is difficult to forecast its place in anaesthetic practice as a stand-alone monitor of blood pressure. This type of monitor should combine other desirable features such as electrocardiographic, pulse oximetry, capnometry and temperature capabilities. Further studies are needed to assess its performance. For the time being and pending future investigations, before embarking on a therapeutic intervention based on a TBP reading, a check of the blood pressure by the oscillometric technique would be advisable. Further engineering improvements are needed and should be pursued to increase the reliability and accuracy of the N-CAT N-500 monitor. At its present level of performance, the N-CAT N-500 should not be taken as a substitute where invasive monitoring is required.

\section{References}

1 Drzewiecki GM, Melbin J, Noordergraaf A. Arterial tonometry: review and analysis. J Biomechanics 1983; 16 : 141-52.

2 Kemmotsu O, Ueda M, Otsuka H, et al. Blood pressure measurement by arterial tonometry in controlled hypotension. Anesth Analg 1991; 73: 54-8.

3 Gardner RM. Direct blood pressure measurement - dynamic response requirements. Anesthesiology 1981; 54: 227-36.

4 Bland JM, Altman DG. Statistical methods for assessing agreement between two methods of clinical measurement. Lancet 1986; 1: 307-10.

5 Gibbs NM, Larach DR, Derr JA. The accuracy of Finapres $(10)$ noninvasive mean arterial pressure measurements in anesthetized patients. Anesthesiology 1991; 74: 647-52.

6 Epstein RH, Bartkowski RR, Huffnagle S. Continuous noninvasive finger blood pressure during controlled hypotension. A comparison with intraarterial pressure. Anesthesiology 1991; 75: 796-803.

7 Aitken HA, Todd JG, Kenny GNC. Comparison of the Finapres and direct arterial pressure monitoring during profound hypotensive anaesthesia. Br J Anaesth 1991; 67: 36-40.

8 Gorback MS, Quill TJ, Lavine $M L$. The relative accuracies of two automated noninvasive arterial pressure measurement devices. J Clin Monit 1991; 7: 13-22.

9 Bardoczky GI, Levarlet $M$, Engelman E, d'Hollander $A$, $S c h m a r t z D$. Continuous noninvasive blood pressure monitoring during thoracic surgery. J Cardiothorac Vasc Anesth 1992; 6: 51-4. 